\title{
Robust control of constrained systems given an information structure
}

\section{Conference Paper}

Author(s):

Furieri, Luca (D); Kamgarpour, Maryam (D)

Publication date:

2017

Permanent link:

https://doi.org/10.3929/ethz-b-000245229

Rights / license:

In Copyright - Non-Commercial Use Permitted

Originally published in:

https://doi.org/10.1109/CDC.2017.8264169 


\title{
Robust Control of Constrained Systems given an Information Structure
}

\author{
Luca Furieri and Maryam Kamgarpour
}

\begin{abstract}
We study finite horizon optimal control where the controller is subject to sensor-information constraints, that is, each input has access to a fixed subset of states at all times. In particular, we consider linear systems affected by exogenous disturbances with state and input constraints. We establish the class of sensor-information structures that allows for the formulation of this optimization problem as a convex program. In the literature, Quadratic Invariance (QI) is a well-established result that is applicable to the infinite horizon unconstrained case. We show that, despite state and inputs constraints being enforced, QI results can be naturally adapted to our problem. To this end, we highlight and exploit the connection between Youla parametrization and disturbance-feedback policies. Additionally, we provide graph-theoretic visual insight which is consistent with Partially Nested (PN) interpretations.
\end{abstract}

\section{INTRODUCTION}

Critical emerging large-scale systems, such as the electric power grids, teams of autonomous vehicles, the internet, economic systems, feature interacting autonomous decision making agents. The agents can be equipped with sensing, computation, communication and actuation capabilities. These applications require study at the intersection between two research topics:

1) Networked control systems; components such as sensors, actuators and computing units are linked through real-time communication channels and have access to different information, usually due to geographic distance or privacy concerns.

2) Efficient design of stabilizing and optimal state-feedback controllers; in scenarios involving constrained large-scale systems, the underlying sensor-information structure, i.e. which component has access to which sensors, hence what state information is available at all given times, has a great impact on the complexity of the control system design.

It has been known since 1968 [1] that the design of optimal controllers with information limitations is in general an NP-hard problem, and the optimal controller can be a nonlinear function of the states. Hence, significant work has been directed to the subclass of affine controllers, subject to special information structures amenable to convex formulations [2]-[4].

All such notions are generalized in [5], where the authors establish a necessary and sufficient condition for tractability of optimal control under information structures, referred to as

This research was gratefully funded by the European Union ERC Starting Grant CONENE.

The authors are with the Automatic Control Laboratory, Department of Information Technology and Electrical Engineering, ETH Zurich, Switzerland. e-mails: \{furieril, mkamgar\}@control.ee.ethz.ch quadratic invariance (QI). Under the QI condition, one can use transfer function approaches to compute solutions for this convex optimization problem [6]. It has been observed that computations at the transfer function level can be sensitive to numerical noise. Moreover, such a representation does not allow for a satisfactory insight into the internal operations of optimal controllers. This motivates study on structured optimal control within state-space representations. In [7], an important effort is done towards this direction. The authors reinterpret the results in [8] in terms of partially ordered sets (Posets). A recent approach, based on the so called System Level Parametrizations (SLP), identifies and relaxes the assumptions implicit to the QI framework so as to strictly broaden the class of distributed control problems solved through convex programming [9], [10]. All the referenced work [7]-[10] consider infinite horizon optimization, without constraints on the states and the inputs.

A successful approach to address state and input constraints is receding horizon control. One line of work has considered rank relaxation approaches to obtain convex approximations of distributed control in finite horizon [11]. Alternatively, distributed model predictive control [12], [13] considers a large system divided into subsystems that are dynamically coupled. The neighbouring subsystems can communicate in order to achieve a local or system-wide goal, while being robust to unknown dynamic couplings. These approaches are readily amenable to many applications, as they include methods to distribute the computations; nonetheless, they do not provide explicit insight on the role of information structures, convexity of the problem and conservativeness of the solution.

The main contribution of this work is the characterization of all convex finite horizon optimal control problems for linear systems subject to state and input constraints, in the presence of a fixed sensor-information structure. The key insight to our approach is the use of disturbance-feedback for robust control of constrained systems [14]. The disturbance-feedback approach in receding horizon control is akin to Youla parametrization in classical control theory. Based on this connection, we then exploit and adapt the results on convexity for infinite horizon distributed control of unconstrained systems [5]. Furthermore, we derive simple conditions to verify QI independent of the horizon length, based on certain properties of the system controllability matrix. We will show the equivalence of these conditions to a graphical condition for QI, consistent with previously established results [4], [15]-[17]. As such, we hope to provide additional insight into the seminal work of [15] and show applicability even in the case in which 
constraints on states and inputs are present.

In Section II, we formulate the finite horizon optimal control problem under study. We review the disturbance feedback approach to enable a convex formulation for the centralized problem. In Section III, we provide the main result about sensor-information structures that enable convexity. Finally, in Section IV, we consider the time varying control of a constrained and unstable 3-dimensional system, whose sensor-information structure is QI. Hence, we solve the proposed convex program and show numerical results.

\section{PROBlem Formulation}

We consider a discrete time system

$$
x_{k+1}=A x_{k}+B u_{k}+w_{k}, \quad \forall k \in \mathbb{Z}_{[0, \infty)},
$$

where $x_{k} \in \mathbb{R}^{n}, u_{k} \in \mathbb{R}^{m}, w_{k} \in \mathcal{W}$ and $\mathcal{W} \subseteq \mathbb{R}^{n}$ is the set of possible disturbances. Throughout, we indicate the $i$-th component of vectors $x_{k}, u_{k}, w_{k}$ at time $k$ as $x_{k}^{i} \in \mathbb{R}$, $u_{k}^{i} \in \mathbb{R}, w_{k}^{i} \in \mathbb{R}$ respectively. The system starts from a known initial condition $x_{0} \in \mathbb{R}^{n}$.

In what follows, predictions of the system's evolution over a finite time horizon of length $N$ will be used to compute appropriate control policies. The states and inputs need to satisfy the following constraints for all $k \in \mathbb{Z}_{[0, N-1]}$ :

$$
\left[\begin{array}{l}
x_{k} \\
u_{k}
\end{array}\right] \in \mathcal{T} \subseteq \mathbb{R}^{n+m}, x_{N} \in \mathcal{X}_{f} \subseteq \mathbb{R}^{n}, \forall w_{k} \in \mathcal{W},
$$

i.e. robustly for all allowable sequences of disturbances. Our goal is to find a history dependent, state-feedback controller $u_{k}=\gamma\left(x_{0}, \cdots, x_{k}\right), \gamma: \mathbb{R}^{n(k+1)} \rightarrow \mathbb{R}^{m}$ such that constraints (2) are satisfied, while minimizing an objective function $J\left(x_{0}, \cdots, x_{N}, u_{0}, \cdots, u_{N-1}\right)$, and with the requirement that $u_{k}(\cdot)$ complies with the sensor-information structure as defined in the following subsection.

The search in the class of all state-feedback policies is, unfortunately, intractable in general. Hence, we restrict to the class of controllers such that $\gamma(\cdot)$ is affine in the history of the states. This is a rich class of state-feedback policies which allows convex formulation of the control problem [14].

The state-feedback controller is subject to sensor-information constraints in the sense that follows.

1) Sensor-information structures and sparsity constraints: Given a matrix $X \in \mathbb{R}^{a \times b}$, we denote throughout as $X(i, j) \in \mathbb{R}$ its element at row $i$ and column $j$. Define the binary matrix $S \in\{0,1\}^{m \times n}$. We state that the control input $u_{k}^{i}$ at time $k$ has access to state $x_{l}^{j}$ for every time $l \in \mathbb{Z}_{[0, k]}$ if and only if $S(i, j)=1$. Hence, the binary matrix $S$ can encode sensor placement for the system. Suppose $X \in\{0,1\}^{a \times b}$, and define the operator

$$
\operatorname{Sparse}(X)=\left\{Y \in \mathbb{R}^{a \times b} \mid Y_{i, j}=0 \forall i, j \text { s.t. } X_{i, j}=0\right\} \text {. }
$$

Then, $\mathcal{S}=\operatorname{Sparse}(S) \subseteq \mathbb{R}^{m \times n}$ is the subspace of linear operators within the sensor-information structure encoded by $S$.

In order to impose the given sensor-information structure in the form of constraints for the optimization problem, for every time instant $k$, we consider the following control policies

$$
\begin{aligned}
& u_{k}=\sum_{j=0}^{k} L_{k, j} x_{j}+g_{k}, \\
& L_{k, j} \in \mathcal{S} \subseteq \mathbb{R}^{m \times n}, g_{k} \in \mathbb{R}^{m}, \forall j \in \mathbb{Z}_{[0, k]} .
\end{aligned}
$$

The constraint $L_{i, j} \in \mathcal{S}$ is referred to as a sparsity constraint, as it defines a fixed zero-pattern for matrices $L_{i, j}$. We then state the optimization problem under study:

\section{Problem 1}

$$
\begin{array}{ll}
\min _{L_{i, j}, g_{i}} J\left(x_{0}, \cdots, x_{N}, u_{0}, \cdots, u_{N-1}\right) \\
\text { s.t. } & (1),(2),(3), \\
& \forall w_{k} \in \mathcal{W}, \forall k \in \mathbb{Z}_{[0, N-1]} .
\end{array}
$$

In the above, the optimization variables are $L_{i, j} \in$ $\mathbb{R}^{m \times n}, g_{i} \in \mathbb{R}^{m}, \forall i \in \mathbb{Z}_{[0, N-1]}, \forall j \in \mathbb{Z}_{[0, i-1]}$. We assume $J(\cdot)$ is a convex function of its arguments, and sets $\mathcal{T}, \mathcal{X}_{f}$ are convex. To make derivations easier, we restrict $\mathcal{T}, \mathcal{X}_{f}$ and $\mathcal{W}$ to be polytopes. In particular, we consider $\mathcal{T}=\{(x, u) \in$ $\left.\left(\mathbb{R}^{n}, \mathbb{R}^{m}\right) \mid C x+D u \leq b\right\}, C \in \mathbb{R}^{s \times n}, D \in \mathbb{R}^{s \times m}, b \in \mathbb{R}^{s}$ and $\mathcal{X}_{f}=\left\{x \in \mathbb{R}^{n} \mid Y x \leq z\right\}, Y \in \mathbb{R}^{r \times n}, z \in \mathbb{R}^{r}$. Without the sparsity constraints, Problem 1 is well-studied within the dynamic optimization and model predictive control literature. Here we recall the disturbance-feedback approach proposed in [14] to derive a convex formulation for Problem 1, in the absence of sparsity constraints.

\section{A. Disturbance Feedback}

It is convenient to define the vectors of stacked variables:

$$
\begin{aligned}
& \mathbf{x}=\left[\begin{array}{lll}
x_{0}^{\top} & \ldots & x_{N}^{\top}
\end{array}\right]^{\top} \in \mathbb{R}^{n(N+1)}, \\
& \mathbf{u}=\left[\begin{array}{lll}
u_{0}^{\top} & \ldots & u_{N-1}^{\top}
\end{array}\right]^{\top} \in \mathbb{R}^{m N}, \\
& \mathbf{w}=\left[\begin{array}{llll}
x_{0}^{\top} & w_{0}^{\top} & \ldots & w_{N-1}^{\top}
\end{array}\right]^{\top} \in \mathbb{R}^{n(N+1)} .
\end{aligned}
$$

Then, the stacked system equations are

$$
\mathbf{x}=\mathbf{B u}+\mathbf{E w}
$$

where matrices $\mathbf{B} \in \mathbb{R}^{n(N+1) \times m N}, \mathbf{E} \in \mathbb{R}^{n(N+1) \times n(N+1)}$ are explicitly written in the appendix. Their derivation is straightforward from recursive application of (1). The control input can be expressed as

$$
\mathbf{u}=\mathbf{L} \mathbf{x}+\mathbf{g}
$$

where $\mathbf{L} \in \mathbb{R}^{m N \times n(N+1)}$ and $\mathbf{g} \in \mathbb{R}^{m N}$ are defined as

$$
\mathbf{L}=\left[\begin{array}{cccc}
L_{0,0} & 0_{m \times n} & \ldots & 0_{m \times n} \\
\cdots & \ldots & \ldots & \ldots \\
L_{N-1,0} & \cdots & L_{N-1, N-1} & 0_{m \times n}
\end{array}\right], \mathbf{g}=\left[\begin{array}{c}
g_{0} \\
\ldots \\
g_{N-1}
\end{array}\right]_{(6)}
$$

The blocks are $L_{i, j} \in \mathbb{R}^{m \times n}, g_{i} \in \mathbb{R}^{m}$ as in (3). Matrix $\mathbf{L}$ is block-lower triangular for causality. In order to impose sparsity constraints in a compact way on matrix $\mathbf{L}$, we need to define the stacked sensor-sparsity subspace $\mathcal{S} \subseteq$ $\mathbb{R}^{m N \times n(N+1)}$ : 


$$
\mathcal{S}=\left[\begin{array}{ccccc}
\mathcal{S} & 0_{m \times n} & \ldots & 0_{m \times n} & 0_{m \times n} \\
\mathcal{S} & \mathcal{S} & \ldots & 0_{m \times n} & 0_{m \times n} \\
\ldots & \ldots & \ldots & \ldots & \ldots \\
\mathcal{S} & \mathcal{S} & \ldots & \mathcal{S} & 0_{m \times n}
\end{array}\right]
$$

where the $\mathcal{S}$ blocks are the sensor-sparsity subspaces for all the $L_{i, j} \mathrm{~s}$ as in (3). Thus, considering (2), (3) and the stacked operators we have just introduced, we define the set of all admissible, state-feedback affine controllers within the given sensor-information structure as:

$\Pi_{N}^{\mathrm{sf}, \mathrm{S}}\left(x_{0}\right)=\left\{\begin{array}{c}(\mathbf{L}, \mathbf{g}) \text { as in }(6), \mathbf{L} \in \mathcal{S} \\ (\mathbf{L}, \mathbf{g}) \mid\left(x_{k}, u_{k}\right) \text { as in }(1),(2),(3) \\ \forall k \in \mathbb{Z}_{[0, N-1]}, \forall w_{0}, \ldots, w_{N-1} \in \mathcal{W}\end{array}\right\}$

As shown in [14], this set is non-convex regardless of the sparsity constraints being linear. However, reparametrization of the controller as a disturbance-feedback affine policy makes it convex. Our goal is to analyze whether the same applies when the controller is required to comply with a fixed sensor-information structure. In what follows, we show why this is not the case.

First, we introduce the disturbance-feedback controller as

$$
\mathbf{u}=\mathbf{M w}+\mathbf{v}
$$

where $\mathbf{M} \in \mathbb{R}^{m N \times n(N+1)}$ and $\mathbf{v} \in \mathbb{R}^{m N}$ are as

$$
\begin{aligned}
& \mathbf{M}=\left[\begin{array}{ccccc}
0_{m \times n} & 0_{m \times n} & \ldots & 0_{m \times n} & 0_{m \times n} \\
M_{1,0} & 0_{m \times n} & \ldots & 0_{m \times n} & 0_{m \times n} \\
\ldots & \ldots & \ldots & \ldots & \ldots \\
M_{N-1,0} & \ldots & M_{N-1, N-2} & 0_{m \times n} & 0_{m \times n}
\end{array}\right], \\
& \mathbf{v}=\left[\begin{array}{lll}
v_{0}^{\top} & \ldots & v_{N-1}^{\top}
\end{array}\right]^{\top} .
\end{aligned}
$$

Notice the particular structure of $\mathbf{M}$, which implies that the control input at time $k$ can only depend on the disturbances up until time $k-1$, which can be easily reconstructed through (1). The blocks are $M_{i, j} \in \mathbb{R}^{m \times n}, v_{i} \in \mathbb{R}^{m}$.

We can establish the mapping between the state-feedback controller (5) and the disturbance-feedback controller (8) as

$$
\begin{aligned}
& \mathbf{M}=\mathbf{L}(\mathbf{I}-\mathbf{B L})^{-1} \mathbf{E}, \\
& \mathbf{v}=\left(\mathbf{I}+\mathbf{L}(\mathbf{I}-\mathbf{B L})^{-1} \mathbf{B}\right) \mathbf{g}, \\
& \mathbf{L}=\mathbf{M}(\mathbf{B M}+\mathbf{E})^{-1}, \\
& \mathbf{g}=\left(\mathbf{I}-\mathbf{M}(\mathbf{B M}+\mathbf{E})^{-1} \mathbf{B}\right) \mathbf{v} .
\end{aligned}
$$

Accordingly, we introduce the set of admissible disturbance-feedback controllers as

$$
\Pi_{N}^{\mathrm{df}, \mathrm{S}}\left(x_{0}\right)=\left\{\begin{array}{c}
(\mathbf{M}, \mathbf{v}) \text { as in }(9) \\
(\mathbf{M}, \mathbf{v}) \mid \begin{array}{l}
F \mathbf{v}+\max _{w_{0}, \ldots, w_{N \cdot 1} \in \mathcal{W}} \\
\mathbf{M}(\mathbf{B M}+\mathbf{E})^{-1} \in \mathcal{S}
\end{array}(F \mathbf{M}+G) \mathbf{w} \leq c
\end{array}\right\}
$$

Matrices $F \in \mathbb{R}^{(N s+r) \times m N}, G \in \mathbb{R}^{(N s+r) \times n(N+1)}, c \in$ $\mathbb{R}^{N s+r}$ capture the state and input constraints (2). For completeness, their explicit form is provided in the appendix.
Finally, we define the set of admissible initial states in both parametrizations as

$$
\begin{aligned}
& \mathcal{X}_{N}^{\mathrm{sf}, \mathrm{S}}=\left\{x_{0} \in \mathbb{R}^{n} \mid \Pi_{N}^{\mathrm{sf}, \mathrm{S}}\left(x_{0}\right) \neq \emptyset\right\}, \\
& \mathcal{X}_{N}^{\mathrm{dff}, \mathrm{S}}=\left\{x_{0} \in \mathbb{R}^{n} \mid \Pi_{N}^{\mathrm{df}, \mathrm{S}}\left(x_{0}\right) \neq \emptyset\right\} .
\end{aligned}
$$

The following theorem then clarifies the link between statefeedback and disturbance-feedback parametrizations.

Theorem 1: $\mathcal{X}_{N}^{\mathrm{df}, \mathrm{S}}=\mathcal{X}_{N}^{\mathrm{sf}, \mathrm{S}}$. Moreover, given an $x_{0} \in \mathcal{X}_{N}^{\mathrm{df}, \mathrm{S}}$, for any $(\mathbf{M}, \mathbf{v}) \in \Pi_{N}^{\mathrm{dff}, \mathrm{S}}\left(x_{0}\right)$ an admissible $(\mathbf{L}, \mathbf{g}) \in \Pi_{N}^{\mathrm{sf}, \mathrm{S}}\left(x_{0}\right)$ can be found such that the resulting state and input trajectories (4), (5) are reproduced for any possible disturbance sequence, and vice-versa.

Proof: By definition, for any $x_{0} \in \mathcal{X}_{N}^{\text {sf,S }}$ there exists a pair $(\mathbf{L}, \mathbf{g}) \in \Pi_{N}^{\mathrm{sf}, \mathrm{S}}\left(x_{0}\right)$. Fix any disturbance sequence $\mathbf{w} \in \mathcal{W}^{N}$ and consider system (4), (5). By plugging the state-feedback $\mathbf{u}=\mathbf{L x}+\mathbf{g}$ into the system equations, we derive $\mathbf{x}=(\mathbf{I}-\mathbf{B L})^{-1}(\mathbf{B g}+\mathbf{E w})$ and plugging this back into (5):

$$
\mathbf{u}=\mathbf{L}(\mathbf{I}-\mathbf{B L})^{-1} \mathbf{E w}+\left(\mathbf{I}+\mathbf{L}(\mathbf{I}-\mathbf{B L})^{-1} \mathbf{B}\right) \mathbf{g} .
$$

Notice that BL is nilpotent, so (I-BL) is always invertible. An equivalent disturbance-feedback controller $(\mathbf{M}, \mathbf{v})$ achieving the same trajectories is obtained by:

$$
\begin{aligned}
& \mathbf{M}=\mathbf{L}(\mathbf{I}-\mathbf{B L})^{-1} \mathbf{E}, \\
& \mathbf{v}=\left(\mathbf{I}+\mathbf{L}(\mathbf{I}-\mathbf{B L})^{-1} \mathbf{B}\right) \mathbf{g} .
\end{aligned}
$$

It is easy to verify $(\mathbf{M}, \mathbf{v}) \in \Pi_{N}^{\mathrm{df}, \mathrm{S}}\left(x_{0}\right)$, so $x_{0} \in \mathcal{X}_{N}^{\mathrm{df}, \mathrm{S}}$.

By definition, for any $x_{0} \in \mathcal{X}_{N}^{\mathrm{df}, \mathrm{S}}$ there exists a pair $(\mathbf{M}, \mathbf{v}) \in \Pi_{N}^{\mathrm{df}, \mathrm{S}}\left(x_{0}\right)$. Fix any disturbance sequence $\mathbf{w} \in \mathcal{W}^{N}$ and consider system (4), (8). By plugging the disturbance-feedback $\mathbf{u}=\mathbf{M w}+\mathbf{v}$ into the system equations, we derive $\mathbf{w}=(\mathbf{B M}+\mathbf{E})^{-1}(\mathbf{x}-\mathbf{B v})$ and plugging this back into (8):

$$
\mathbf{u}=\mathbf{M}(\mathbf{B M}+\mathbf{E})^{-1}(\mathbf{x}-\mathbf{B v})+\mathbf{v} .
$$

So an equivalent state-feedback controller $(\mathbf{L}, \mathbf{g})$ achieving the same trajectories is obtained by:

$$
\begin{aligned}
& \mathbf{L}=\mathbf{M}(\mathbf{B M}+\mathbf{E})^{-1}, \\
& \mathbf{g}=\left(\mathbf{I}-\mathbf{M}(\mathbf{B M}+\mathbf{E})^{-1} \mathbf{B}\right) \mathbf{v} .
\end{aligned}
$$

It is easy to verify $(\mathbf{L}, \mathbf{g}) \in \Pi_{N}^{\mathrm{sf}, \mathrm{S}}\left(x_{0}\right)$, so $x_{0} \in \mathcal{X}_{N}^{\mathrm{sf}, \mathrm{S}}$.

Note that our mappings are slightly different than the ones in [14], due to the inclusion of $x_{0}$ in the extended stacked disturbance $\mathbf{w}$.

\section{B. Equivalent parametrizations}

The idea of using disturbance-feedback in control theory is not new. In fact, Youla parametrization, a fundamental approach in characterizing stabilizing controllers, hinges on this idea. Let us briefly recall this approach.

Consider a proper transfer function

$$
P(s)=\left[\begin{array}{ll}
P_{z w}(s) & P_{z u}(s) \\
P_{y w}(s) & P_{y u}(s)
\end{array}\right],
$$

which describes the dynamic relationships from the disturbance signal $w(s)$ and the input signal $u(s)$ to the objective 
variables signal $z(s)$ and the output signal $y(s)$. Connect the plant $P(s)$ in closed loop with a linear output feedback controller $K(s)$, whose transfer function from $y(s)$ to $u(s)$ is proper. The resulting map from $w(s)$ to $u(s)$ is

$$
M(s)=K(s)\left[I-P_{y u}(s) K(s)\right]^{-1} P_{y w}(s) .
$$

In the Youla parametrization literature, the Youla parameter is defined as $Q(s)=K(s)\left[I-P_{y u}(s) K(s)\right]^{-1}$. One can observe that our disturbance-feedback controller in equation (14) is the equivalent transformation in finite horizon. In particular, $\mathbf{M}, \mathbf{E}$ and $\mathbf{M} \mathbf{E}^{-1}$ correspond to $M(s), P_{y w}(s)$ and $Q(s)$ respectively. This connection motivates us to exploit the convexity results for distributed controllers in the infinite dimensional and unconstrained setting [8].

\section{CONDITIONS FOR CONVEXITY}

Motivated by [5], [18], we first introduce the following definitions and lemmas.

Definition 1 (Closed-loop map): We define the closed-loop map function $h: \mathbb{R}^{m N \times n(N+1)} \times$ $\mathbb{R}^{n(N+1) \times m N} \rightarrow \mathbb{R}^{m N \times n(N+1)}$ as $h(\mathbf{X}, \mathbf{Y})=-\mathbf{X}(\mathbf{I}-\mathbf{Y X})^{-1}$. Also, we introduce the set $h(\mathcal{S}, \mathbf{B})=\{h(\mathbf{L}, \mathbf{B}), \forall \mathbf{L} \in \mathcal{S}\}$. According to Section II-B and (14), the set $h(\mathcal{S}, \mathbf{B})$ can be interpreted as the set of Youla parameters that comply with the given information structure.

Definition 2 (Quadratic Invariance, from [5]): Let $K_{1}, K_{2}: \mathbb{R}^{a} \rightarrow \mathbb{R}^{b}, G: \mathbb{R}^{b} \rightarrow \mathbb{R}^{a}$ be finite-dimensional linear maps. Let $K_{1}, K_{2} \in \tilde{S} \subseteq \mathbb{R}^{b \times a}$, where $\tilde{S}=\operatorname{Sparse}(\mathcal{K})$ for some $\mathcal{K} \in\{0,1\}^{b \times a}$. Then, $\tilde{S}$ is Quadratically Invariant (QI) with respect to $G$ if $K_{1} G K_{2} \in \widetilde{S}, \forall K_{1}, K_{2} \in \tilde{S}$.

Note that the general definition of QI presented in other works, involves infinite-dimensional linear operators [5], [8].

Recall from the appendix the definitions of the system stacked dynamic matrix $\mathbf{B}$ and of the stacked sensor-sparsity subspace $\mathcal{S}$. We introduce the following two key lemmas.

Lemma 1: The set $h(\mathcal{S}, \mathbf{B})$ is convex if and only if it is equal to $\mathcal{S}$.

Lemma 1, whose proof is provided in [18], motivates to look for conditions so that $h(\mathcal{S}, \mathbf{B})=\mathcal{S}$.

Lemma 2: $h(\mathcal{S}, \mathbf{B})=\mathcal{S} \Longleftrightarrow \mathcal{S}$ is $Q I$ w.r.t. B .

One of the key insights into proving Lemma 2 is the power series expansion of the closed-loop loop map $h(\mathbf{X}, \mathbf{Y})$. To ensure convergence of the power series, additional assumptions for plant and controller are needed in the infinite dimensional setting: see Section III-A in [5]. Within the class of finite-dimensional operator we consider, convergence conditions are satisfied by construction. Indeed, matrix BL that appears in $h(\mathbf{L}, \mathbf{B})$ has a null diagonal for any choice of the dynamical system and the affine controller, hence it is nilpotent.

Lemma 1 and 2 above are particular cases of Theorem 14 in [5] and Theorem 5 in [18], respectively. Now we present our main result on the application of the above to characterization of feasible disturbance-feedback policies.

Theorem 2: The set $\Pi_{N}^{\mathrm{df}, \mathrm{S}}\left(x_{0}\right)$ is convex if and only if $\mathcal{S}$ is QI with respect to $\mathbf{B}$. In this case, the sparsity constraint on $\mathbf{M}$ can be equivalently expressed as $\mathbf{M E}^{-1} \in \mathcal{S}$.
Proof:

Define the set of disturbance-feedback matrices that satisfy sparsity constraints as

$$
\mathcal{C}=\left\{\mathbf{M} \mid \mathbf{M}=\mathbf{L}(\mathbf{I}-\mathbf{B L})^{-1} \mathbf{E}, \forall \mathbf{L} \in \mathcal{S}\right\} .
$$

Then, by definition, it holds that $\mathcal{C}=-h(\mathcal{S}, \mathbf{B}) \mathbf{E}$. As matrix $\mathbf{E}$ is always invertible (the eigenvalues are all equal to 1 ), it follows by direct application of Lemma 1 and Lemma 2 that $\mathcal{C}$ is convex if and only if $\mathcal{S}$ is QI with respect to $\mathrm{B}$. This is equivalent to saying that the set of matrices $\mathbf{M}$ satisfying sparsity constraints is convex if and only if $\mathcal{S}$ is QI with respect to $\mathbf{B}$. As the remaining constraints in $\Pi_{N}^{\mathrm{df}, \mathrm{S}}\left(x_{0}\right)$ are linear in the decision variables $\mathbf{M}$, then $\Pi_{N}^{\mathrm{df}, \mathrm{S}}\left(x_{0}\right)$ is convex if and only if $\mathcal{S}$ is QI with respect to $\mathbf{B}$.

Now notice that $\mathbf{M}(\mathbf{B M}+\mathbf{E})^{-1}=-h\left(\mathbf{M E}^{-1},-\mathbf{B}\right)$. From Lemma 2, it holds

$$
\mathcal{S} \text { is QI w.r.t. } \mathbf{B} \Longleftrightarrow\left\{h\left(\mathbf{M E}^{-1}, \mathbf{B}\right) \mid \mathbf{M E}^{-1} \in \mathcal{S}\right\}=\mathcal{S} \text {, }
$$

and by set equivalence:

$$
\begin{aligned}
& \left\{h\left(\mathbf{M} \mathbf{E}^{-1}, \mathbf{B}\right) \mid \mathbf{M} \mathbf{E}^{-1} \in \mathcal{S}\right\}=\mathcal{S} \\
& \forall \mathbf{M} \mathbf{E}^{-1} \in \mathcal{S},-h\left(\mathbf{M} \mathbf{E}^{-1},-\mathbf{B}\right) \in \mathcal{S} \\
& \text { and } \forall-h\left(\mathbf{M} \mathbf{E}^{-1},-\mathbf{B}\right) \in \mathcal{S}, \mathbf{M E}^{-1} \in \mathcal{S},
\end{aligned}
$$

in which we also used the fact that QI w.r.t. B implies QI w.r.t. -B. Hence, we can replace the sparsity constraints in $\Pi_{N}^{\mathrm{df}, \mathrm{S}}\left(x_{0}\right)$ with $\mathrm{ME}^{-1} \in \mathcal{S}$ if and only if $\mathcal{S}$ is QI with respect to $\mathbf{B}$.

By direct application of Theorem 2, under the assumption of QI, we formulate the disturbance-feedback convex program which is equivalent to Problem 1.

\section{Problem 2}

$$
\begin{array}{ll}
\min _{\mathbf{M}, \mathbf{v}} J(\mathbf{x}, \mathbf{u}) \\
\text { s.t. } \quad \mathbf{x} \text { as in }(4), \mathbf{u} \text { as in }(8),(\mathbf{M}, \mathbf{v}) \text { as in }(9), \\
& F \mathbf{v}+\max _{w_{0}, \ldots, w_{N-1} \in \mathcal{W}}(F \mathbf{M}+G) \mathbf{w} \leq c, \\
& \mathbf{M E}^{-1} \in \mathcal{S} .
\end{array}
$$

The optimal solution $\left(\mathbf{M}^{\star}, \mathbf{v}^{\star}\right)$ to Problem 2 can be translated into the optimal solution $\left(\mathbf{L}^{\star}, \mathbf{g}^{\star}\right)$ of Problem 1 through the mapping highlighted in Theorem 1 .

In summary, we see that our setup generalizes [8] to include state and input constraints in finite horizon. Also, it generalizes [14] to include sparsity constraints. At the same time, however, our convexity results can be interpreted as a special case of those in [5] with a restriction to finite dimensional linear maps.

Remark 1: It has been shown in [15] that the notions of partially nested (PN) and QI information structures are equivalent. Hence, Proposition 1 and Proposition 2 in [19] can be regarded as equivalent interpretation of the result in Theorem 2. However, our proof method is different, as it is based on QI results over finite-dimensional operators and analysis of the closed loop operator $h(\cdot)$. This allows for certification of necessity and sufficiency of QI for convexity, whereas Proposition 1 and Proposition 2 in [19] only show sufficiency of PN for convexity. 


\section{A. Interpreting QI sensor-information structures.}

Given the importance of QI for convex formulation of structured controllers, it is necessary to investigate their control-theoretic and graphical meaning. Our first result identifies a condition for QI, defined based on the columns of the system controllability matrix $\left[\begin{array}{lllll}B & A B & \ldots & A^{n-1} B\end{array}\right]$.

Theorem 3: The following two statements are equivalent:

a) $\mathcal{S}$ is QI with respect to $\mathrm{B}$.

b) $\mathcal{S}$ is QI with respect to $\left(A^{s} B\right), \quad \forall s \in \mathbb{Z}_{[0, n-1]}$.

Proof: b) $\Longrightarrow$ a). For $\mathbf{L}, \mathbf{L}^{\prime} \in \mathcal{S}$, define $\Phi=\mathbf{L} \mathbf{B L}^{\prime}$. Divide $\Phi$ into a $N \times(N+1)$ block matrix, each block with dimension $m \times n$, and reference its $(i, j)$ block as $\Phi_{i, j} \in$ $\mathbb{R}^{m \times n}$. Then, considering the blocks of $\mathbf{L}, \mathbf{L}^{\prime}$ as in (6),

$$
\Phi_{i, j}=\sum_{h=0}^{i-1}\left[\sum_{k=0}^{i-h-1}\left(L_{i, h+k+1} A^{k} B\right) L_{h, j}^{\prime}\right] .
$$

Recall $L_{a, b}=0_{m \times n}$ whenever $b>a$. From (20) we see that every block $\Phi_{i, j}$ is the sum of subterms in the form $L A^{s} B L^{\prime}$, where $L, L^{\prime} \in \mathcal{S}$ are generic $m \times n$ blocks of $\mathbf{L}$ and $\mathbf{L}^{\prime}$ respectively, and $s \in \mathbb{Z}_{[0, N-1]}$. A sufficient condition to ensure $\Phi \in \mathcal{S}$, recalling the Cayley Hamilton theorem, is then:

$$
L\left(A^{s} B\right) L^{\prime} \in \mathcal{S} \quad \forall L, L^{\prime} \in \mathcal{S}, \forall s \in \mathbb{Z}_{[0, n-1]},
$$

i.e. $S$ is QI with respect to $\left(A^{s} B\right), \forall s \in \mathbb{Z}_{[0, n-1]}$. Hence b) $\Longrightarrow$ a). Next, we prove a) $\Longrightarrow$ b) by contrapositive. If b) is false, then $\exists L_{1}, L_{2} \in \mathcal{S}$ and $s^{\star} \in \mathbb{Z}_{[0, n-1]}$ such that $L_{1} A^{s^{\star}} B L_{2} \notin S$. We construct a $\mathbf{L}^{\star} \in \mathcal{S}$ such that $\mathbf{L}^{\star} \mathbf{B} \mathbf{L}^{\star} \notin \mathcal{S}$. Choose the $m \times n$ blocks of $\mathbf{L}^{\star} \in \mathcal{S}$ as

$$
L_{i, j}^{\star}= \begin{cases}L_{1} & \text { if } i=j=s^{\star}+1 \\ L_{2} & \text { if } i=j=0 \\ 0 & \text { otherwise }\end{cases}
$$

and $\Phi^{\star}=\mathbf{L}^{\star} \mathbf{B} \mathbf{L}^{\star}$. It is easy to verify that $\Phi_{s^{\star}+1,0}^{\star}=$ $L_{s^{\star}+1, s^{\star}+1}^{\star} A^{s^{\star}} B L_{0,0}^{\star}=L_{1} A^{s^{\star}} B L_{2} \notin S$. Hence $\Phi^{\star} \notin \mathcal{S}$.

Next, we exploit Theorem 3 to establish a graph-theoretic interpretation of QI for sensor-information structures. Given the system matrices $(A, B)$ as in (1), define the graph $\mathcal{G}=$ $(\mathcal{V}, \mathcal{E})$, where $\mathcal{V}=\left\{\nu_{x_{1}}, \ldots, \nu_{x_{n}}, \nu_{u_{1}}, \ldots, \nu_{u_{m}}\right\}$ is the set of nodes associated with the $n$ states and the $m$ inputs, and we denote as $\varepsilon_{\nu_{a}, \nu_{b}}$ an arc in $\mathcal{E}$ with tail in $\nu_{a} \in \mathcal{V}$ and head in $\nu_{b} \in \mathcal{V}$. For every $s \in \mathbb{Z}_{[0, n-1]}$, define $\Delta_{s} \in \mathbb{R}^{n \times m}$ so that $\Delta_{s}(i, j)=1$ if $\left(A^{s} B\right)(i, j) \neq 0$ and $\Delta_{s}(i, j)=0$ otherwise. Let the nonzero entries in $A$ encode the arcs $\varepsilon_{\nu_{x_{i}}, \nu_{x_{j}}}$, the nonzero entries in $B$ encode the arcs $\varepsilon_{\nu_{u_{k}}, \nu_{x_{l}}}$, the nonzero entries in matrix $S$ encode the arcs $\varepsilon_{\nu_{x_{r}}, \nu_{u_{s}}}$, for $i, j, l, r \in$ $\mathbb{Z}_{[1, n]}$ and $k, s \in \mathbb{Z}_{[1, m]}$. One can then exploit Theorem 3 to derive the following graph-theoretic interpretation of QI.

Corollary 1: The following statements are equivalent:

1) $\mathcal{S}$ is $\mathrm{QI}$ with respect to $\left(A^{s} B\right), \quad \forall s \in \mathbb{Z}_{[0, n-1]}$.

2) Every time there is a walk of length $s+1$ from $\nu_{u_{j}}$ to $\nu_{x_{i}}$ and $\epsilon_{\nu_{x_{i}}, \nu_{u_{k}}} \in \mathcal{E}$, and additionally $\Delta_{s}(i, j)=1$, then for every $l$ such that $\varepsilon_{\nu_{x_{l}}, \nu_{u_{j}}} \in \mathcal{E}$, also $\varepsilon_{\nu_{x_{l}}, \nu_{u_{k}}} \in \mathcal{E}$.

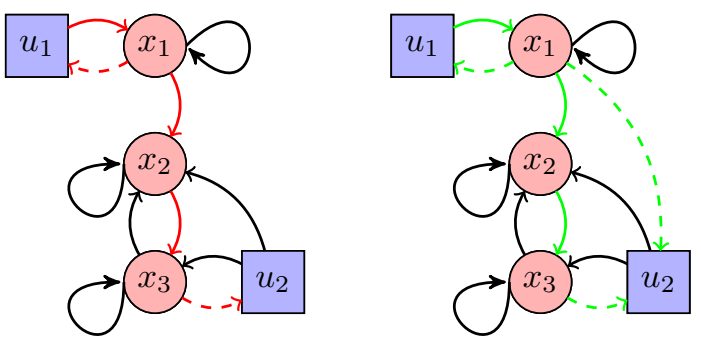

Fig. 1. Red and green lines indicate critical walks for QI. The dashed lines show the sensor-information structure. In the left graph, we can identify two coupled subsystems. The first one, with state $x_{1}$ is accessed and controlled by $u_{1}$. The second one, with states $x_{2}, x_{3}$, is accessed and controlled by $u_{2}$. Because of the coupling $x_{1} \rightarrow x_{2}$, the decisions of $u_{1}$ influence those of $u_{2}$ after 3 time steps. However $u_{2}$ does not have direct access to $x_{1}$, hence the structure is not QI and the problem is nonconvex. By adding the edge $x_{1} \rightarrow u_{2}$, we get a QI structure.

Proof: Consider the statement

$$
S(k, i) \Delta_{s}(i, j) S(j, l)(1-S(k, l))=0, \forall i, j, l, m, s,
$$

where $\Delta_{s}(i, j)=1$ if $\left(A^{s} B\right)(i, j) \neq 0$ and $\Delta_{s}(i, j)=0$ otherwise. First, we show that $(21)$ is equivalent to statement 1). For 1$) \Longrightarrow(21)$, we reason by contrapositive. Suppose $S(k, l)=0, \Delta_{s}(i, j)=1, L, L^{\prime} \in S$, but $L(k, i) L^{\prime}(j, l) \neq 0$, which implies $\mathcal{S}(k, i)=\mathcal{S}(j, l)=1$. Set all the other elements in $L, L^{\prime}$ to zero. Then $\left(L \Delta_{s} L^{\prime}\right)(k, l)=$ $L(k, i) \Delta_{s}(i, j) L^{\prime}(j, l) \neq 0$, which contradicts QI. For $(21)$ $\Longrightarrow 1$ ), notice that (21) implies $L(k, i)=0$ or $L^{\prime}(j, l)=0$ whenever $L, L^{\prime} \in \mathcal{S}, \mathcal{S}(k, l)=0$ and $\Delta_{s}(i, j)=1$, which implies $\left(L \Delta_{s} L^{\prime}\right)(k, l)=\sum_{i} \sum_{j} L(k, i) \Delta_{s}(i, j) L^{\prime}(j, l)=0$ for any such case and hence 1).

Last, we show (21) is equivalent to statement 2). Notice that (21) is violated if and only if $S(k, l)=0$ while all the rest is one. Graphically, this would mean that state $x_{l}$ is not observed by input $u_{k}$. At the same time, $S(j, l)=1$, $\Delta_{s}(i, j)=1$ and $S(k, i)=1$ can be respectively interpreted as $x_{l}$ is observed by $u_{j}, u_{j}$ influences $x_{i}$ in $s+1$ time-steps and $x_{i}$ is observed by $u_{k}$. Hence, in accordance with (21), the graphical-condition states that for every such situation, a path from $x_{l}$ to $u_{k}$ must exist.

The corollary above is consistent with the notion of PN information structures. Other works that include the study of PN and their graphical interpretation are [16], [17].

In Figure 1, we show a sensor-information structure which is easily checked not to be QI through the proposed graph-theoretic interpretation. Next to it, we show the corresponding modified QI graph.

\section{Simulations}

We illustrate the proposed approach through a numerical example on an unstable 3-dimensional system. The system 
dynamics are given by

$$
A=\left[\begin{array}{ccc}
1.192 & 0 & 0 \\
0.3 & 0.1 & 0.4 \\
0 & 0.01 & 1.2
\end{array}\right], \quad B=\left[\begin{array}{cc}
0.255 & 0 \\
0 & 0.34 \\
0 & 0.64
\end{array}\right] .
$$

The sensor-information matrix is defined as $S=\left[\begin{array}{lll}1 & 0 & 0 \\ 1 & 0 & 1\end{array}\right]$, and the corresponding sparsity subspace is $\mathcal{S}=\operatorname{Sparse}(S)$. Notice that this system can be described by the QI graph in Figure 1 . The disturbance set $\mathcal{W}$ is chosen as a cube in $\mathbb{R}^{3}$ centered in the origin, edges of length 0.2 and parallel to the cartesian axes. The state constraints are chosen as a cube in $\mathbb{R}^{3}$ centered on the origin, edges of length 6 and parallel to the cartesian axes. The input constraints are chosen as a square in $\mathbb{R}^{2}$ centered on the origin, edges of length 6.4 and parallel to the cartesian axes. The initial state is $x_{0}=$ $2.8\left[\begin{array}{lll}1 & -1 & 1\end{array}\right]^{\top}$ and the prediction horizon is set to $N=8$ time steps.

We select the quadratic cost $J(\mathbf{x}, \mathbf{u})=\hat{\mathbf{x}}^{\top} \mathcal{Q} \hat{\mathbf{x}}+\hat{\mathbf{u}}^{\top} \mathcal{R} \hat{\mathbf{u}}$, where $\mathcal{Q}=I_{n(N+1)}, \mathcal{R}=I_{m N}$ are identity matrices of appropriate dimensions, and $\hat{\mathbf{x}}=\mathbf{B} \hat{\mathbf{u}}+\mathbf{E}\left[\begin{array}{llll}x_{0}^{\top} & 0 & \cdots & 0\end{array}\right]^{\top}$, $\hat{\mathbf{u}}=\mathbf{L} \hat{\mathbf{x}}+\mathbf{g}$ are the disturbance-free trajectories.

In order to ensure that state and input constraints are satisfied for all time instants $k \in \mathbb{Z}_{[0, \infty)}$, we consider the following time varying affine disturbance-feedback policy

$$
u_{k}= \begin{cases}g_{k}+\sum_{j=0}^{k} L_{k, j} x_{j} & \text { if } k \in \mathbb{Z}_{[0, N-1]}, \\ K x_{k} & \text { if } k \in \mathbb{Z}_{[N, \infty)} .\end{cases}
$$

The main challenge is to find a matrix $K$ and a corresponding final set $\mathcal{X}_{f}$ such that $\mathcal{X}_{f}$ is robustly positive invariant under $u=K x$. This is in accordance with Assumption 1 in [14]. Our approach was to first select $K \in \mathcal{S}$ so that all eigenvalues of $(A+B K)$ lie inside the unitary disc and that the trace of $\|(A+B K)\|$ is minimized. To this end, a simple linear program (LP) can be cast, for example, by exploiting stability conditions based on the Gershgorin circles theorem [20]. Thus, we found the stabilizing matrix $K=-\left[\begin{array}{ccc}4.6745 & 0 & 0 \\ 0 & 0 & 1.875\end{array}\right]$, which lies in subspace $S$. Second, we applied the algorithm in [21] to find a robustly invariant approximation of the minimal robustly invariant. Upon verification of $\left(\mathcal{X}_{f}, K \mathcal{X}_{f}\right) \subseteq \mathcal{T}$, we set $\mathcal{X}_{f}$ equal to this approximation.

Last, we solved the optimization problem to control the system in the time interval $[0, N-1]$. As we chose $\mathcal{W}$ to be a polytope, we could translate Problem 2 into an explicit LP. We solved the resulting LP using the MATLAB interface YALMIP with the solver QUADPROG [22], [23]. Since the chosen structure can be easily checked to be QI (e.g. through the graph-theoretic test), the recovered state-feedback controller $\left(\mathbf{L}^{\star}, \mathbf{g}^{\star}\right)$ lies in $\mathcal{S}$. We simulated the system, controlled according to (22), with the disturbance sequence:

$$
\begin{cases}w_{k}^{i}=0.1, & \forall i \in \mathbb{Z}_{[0, n-1]}, k \in \mathbb{Z}_{[0, N-1]}, \\ w_{k}^{i}=U([-0.1,0.1]), & \forall i \in \mathbb{Z}_{[0, n-1]}, k \in \mathbb{Z}_{[N, \infty)},\end{cases}
$$

where $U(\cdot)$ is the uniform distribution between values -0.1 and 0.1. In Figure 2 we report the resulting states trajectories. We can verify that the states reach $\mathcal{X}_{f}$ within 8 time steps and stay there despite random disturbances.

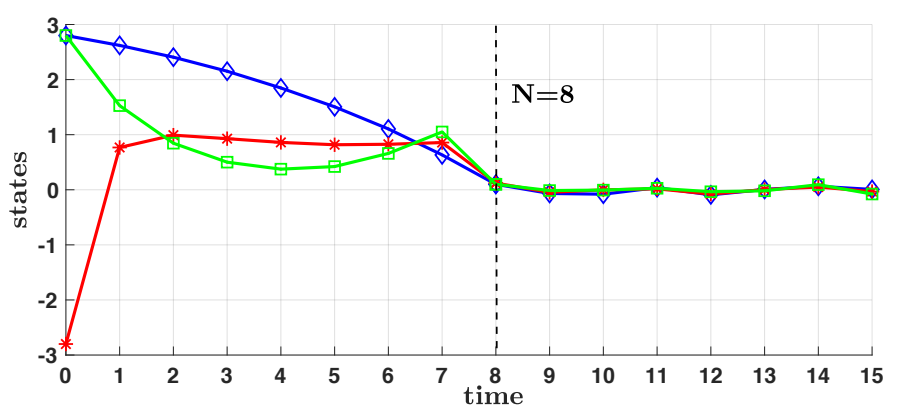

Fig. 2. The state and disturbance trajectories are reported according to the following symbol map. diamond for $x_{k}^{1}$, star for $x_{k}^{2}$, square for $x_{k}^{3}$.

\section{CONCLUSIONS}

We provided a convex program to solve the optimal control problem for constrained linear systems in finite horizon, when a fixed sensor-information structure is enforced. Our result is based on the connection between the Youla parametrization and disturbance feedback policies. In particular, we characterized the class of sensor-information structures that allow for convex optimization. We described their graphical meaning in Theorem 3 and Corollary 1. The applicability of the found results was shown in numerical simulations.

Future work will involve approaches to distribute the centralized computation. Furthermore, it will be interesting to explore convexification approaches for information structures which are not QI.

\section{APPENDIX I \\ NOTATION}

Here we define the following matrices and vectors. The operator $\otimes$ is the Kronecker product. For any matrix $X \in \mathbb{R}^{a \times b}$, $X_{[m, n],[g, h]} \in \mathbb{R}^{(n-m+1) \times(h-g+1)}$ represents the submatrix obtained by picking the rows from $m$ to $n$ and the columns from $g$ to $h$.

$$
\begin{aligned}
\mathbf{E} & =\left[\begin{array}{cccc}
I_{n} & 0 & \ldots & 0 \\
A & I_{n} & \ldots & 0 \\
\ldots & \ldots & \ldots & \ldots \\
A^{N-1} & A^{N-2} & \ldots & I_{n}
\end{array}\right] \in \mathbb{R}^{n(N+1) \times n(N+1)} \\
\mathbf{B} & =\mathbf{E}_{[1, n(N+1)],[n+1, n(N+1)]}\left(I_{N} \otimes B\right) \in \mathbb{R}^{n(N+1) \times m N} \\
\mathbf{C} & =\left[\begin{array}{cc}
I_{N} \otimes C & 0 \\
0 & Y
\end{array}\right] \in \mathbb{R}^{(N s+r) \times n(N+1)} \\
\mathbf{D} & =\left[\begin{array}{c}
I_{N} \otimes D \\
0
\end{array}\right] \in \mathbb{R}^{(N s+r) \times m N} \\
F & =\mathbf{C B}+\mathbf{D} \in \mathbb{R}^{(N s+r) \times m N} \\
G & =\mathbf{C E} \in \mathbb{R}^{(N s+r) \times n(N+1)} \\
c & =\left[\begin{array}{c}
\mathbf{1}_{N} \otimes b \\
z
\end{array}\right] \in \mathbb{R}^{(N s+r)}
\end{aligned}
$$




\section{ACKNOWLEDGMENT}

We acknowledge Paul Goulart for helpful discussions.

\section{REFERENCES}

[1] H. S. Witsenhausen, "A counterexample in stochastic optimum control," SIAM Journal on Control, vol. 6, no. 1, pp. 131-147, 1968.

[2] C.-H. Fan, J. L. Speyer, and C. R. Jaensch, "Centralized and decentralized solutions of the linear-exponential-gaussian problem," IEEE Transactions on Automatic Control, vol. 39, no. 10, pp. 1986-2003, 1994.

[3] P. G. Voulgaris, "A convex characterization of classes of problems in control with specific interaction and communication structures," in American Control Conference, 2001. Proceedings of the 2001, vol. 4. IEEE, 2001, pp. 3128-3133.

[4] K.-C. Chu, "Team decision theory and information structures in optimal control problems." DTIC Document, Tech. Rep., 1971.

[5] M. Rotkowitz and S. Lall, "A characterization of convex problems in decentralized control," IEEE Transactions on Automatic Control, vol. 51, no. 2, pp. 274-286, 2006.

[6] A. Alavian and M. C. Rotkowitz, "Q-parametrization and an sdp for h-optimal decentralized control," IFAC Proceedings Volumes, vol. 46, no. 27 , pp. 301-308, 2013.

[7] P. Shah and P. A. Parrilo, "Optimal decentralized control over posets: A state-space solution for state-feedback," IEEE Transactions on Automatic Control, vol. 58, no. 12, pp. 3084-3096, 2013.

[8] M. C. Rotkowitz, "Parametrization of all stabilizing controllers subject to any structural constraint," in Decision and Control (CDC), 2010. 49th IEEE Conference on. IEEE, 2010, pp. 108-113.

[9] Y.-S. Wang, N. Matni, and J. C. Doyle, "System level parameterizations, constraints and synthesis," 2017.

[10] —_ "Localized lqr optimal control," in Decision and Control (CDC), 2014 IEEE 53rd Annual Conference on. IEEE, 2014, pp. 1661-1668.

[11] G. Fazelnia, R. Madani, A. Kalbat, and J. Lavaei, "Convex relaxation for optimal distributed control problems," IEEE Transactions on Automatic Control, vol. 62, no. 1, pp. 206-221, 2017.

[12] C. Conte, C. N. Jones, M. Morari, and M. N. Zeilinger, "Distributed synthesis and stability of cooperative distributed model predictive control for linear systems," Automatica, vol. 69, pp. 117-125, 2016.

[13] P. Giselsson and A. Rantzer, "On feasibility, stability and performance in distributed model predictive control," IEEE Transactions on Automatic Control, vol. 59, no. 4, pp. 1031-1036, 2014.

[14] P. J. Goulart, E. C. Kerrigan, and J. M. Maciejowski, "Optimization over state feedback policies for robust control with constraints," Automatica, vol. 42, no. 4, pp. 523-533, 2006.

[15] M. Rotkowitz, "On information structures, convexity, and linear optimality," in Decision and Control, 2008. CDC 2008. 47th IEEE Conference on. IEEE, 2008, pp. 1642-1647.

[16] J. Swigart and S. Lall, "A graph-theoretic approach to distributed control over networks," in CDC/CCC 2009. Proceedings of the 48th IEEE Conference on. IEEE, 2009, pp. 5409-5414.

[17] A. Nayyar and L. Lessard, "Structural results for partially nested lqg systems over graphs," in American Control Conference (ACC), 2015. IEEE, 2015, pp. 5457-5464.

[18] L. Lessard and S. Lall, "Quadratic invariance is necessary and sufficient for convexity," in American Control Conference (ACC), 2011. IEEE, 2011, pp. 5360-5362.

[19] W. Lin and E. Bitar, "Performance bounds for robust decentralized control," in American Control Conference (ACC), 2016. IEEE, 2016, pp. $4323-4330$.

[20] S. A. Gershgorin, "Uber die abgrenzung der eigenwerte einer matrix," . , no. 6, pp. 749-754, 1931.

[21] S. V. Rakovic, E. C. Kerrigan, K. I. Kouramas, and D. Q. Mayne, "Invariant approximations of the minimal robust positively invariant set," IEEE Transactions on Automatic Control, vol. 50, no. 3, pp. 406410, 2005.

[22] MATLAB, version (R2015b). Natick, Massachusetts: The MathWorks Inc., 2015.

[23] J. Löfberg, "Yalmip : A toolbox for modeling and optimization in matlab," in In Proceedings of the CACSD Conference, Taipei, Taiwan, 2004. 